\title{
High energy sources during the re-ionization epoch of the universe
}

\section{Felix Mirabel*}

Commissariat à l'Energie Atomique et Energies Alternatives Saclay

E-mail: felix.mirabelecea.fr

\begin{abstract}
The cosmic dark ages are one of the major frontiers in cosmology. So far, most models of the reionization have considered the ultraviolet radiation from massive stars formed in the first galaxies. I will show that high energy sources of X-rays and relativistic jets determined the early thermal history of the universe and maintained it ionized over large volumes of space until the first billon years of the universe, when feedback from super-massive black holes (AGN) took over. High energy sources from compact stellar objects had a direct impact on the properties of the faintest galaxies at high redshifts, the smallest dwarf galaxies in the local universe, and on the cold dark matter model of the universe.
\end{abstract}

Frontiers of Fundamental Physics 14 - FFP14,

15-18 July 2014

Aix Marseille University (AMU) Saint-Charles Campus, Marseille

\footnotetext{
*Speaker.
} 